\title{
Injective mesenchymal stem cell-based treatments for knee osteoarthritis: from mechanisms of action to current clinical evidences
}

\author{
Silvia Lopa ${ }^{1} \cdot$ Alessandra Colombini $^{2} \cdot$ Matteo Moretti $^{1,3,4} \cdot$ Laura de Girolamo $^{2}$ (I)
}

Received: 21 March 2018 / Accepted: 14 August 2018 / Published online: 29 August 2018

(c) The Author(s) 2018

\begin{abstract}
Purpose Osteoarthritis (OA) represents a relevant social and economic burden worldwide. "Mesenchymal stem cells" or, as recently proposed, "medicinal signaling cells" (MSCs) have been recently introduced as injective treatments for OA with the aim of restoring joint homeostasis. The aim of this review is to provide the reader with the tools necessary to interpret the currently available clinical data, focusing on the MSC mechanisms of action which might help to clarify what we should expect from this treatment.

Methods Clinical studies reporting MSC injections for the treatment of knee OA, either freshly isolated or culture-expanded cells, have been included and commented in relation to the supposed therapeutic effect that MSCs might exert giving their supposed mode of actions.

Results The majority of the studies reports significant improvements in terms of pain and knee function compared to baseline values, up to 24 months of follow-up. Although these data support the expected therapeutic effect of this therapy giving the features of these cells, only $14 \%$ of the studies present a control group and more than one-third of them report the results on less than ten patients.

Conclusions Despite the constant presence of positive and satisfactory results in the studies analyzed, the complexity of MSC metabolism and related therapeutic effects as well as the weakness of most of the studies do not allow withdrawing definitive conclusions about the superiority of one tissue source over another, as well as about the best cell dose and the long-term durability of the effects of these procedures. Given the high potential value of these therapies in the treatment of OA, further studies accurately designed, carefully defining the type of patients to be included and pursuing minimal standard requirements in terms of follow-up, number of patients, and types of measurements should be conducted to finally assess the efficacy of MSC-based injective treatments.
\end{abstract}

Keyword Osteoarthritis · Inflammation · Mesenchymal stem cells · Intra-articular injection · Articular cartilage · Bone marrow concentrate $\cdot$ Stromal vascular fraction $\cdot$ Adipose tissue

\section{Abbreviations}

OA Osteoarthritis

MSC Mesenchymal stem cell

BMSCs Bone marrow-derived mesenchymal stem cell

Silvia Lopa and Alessandra Colombini have equally contributed to this work.

Laura de Girolamo

laura.degirolamo@grupposandonato.it

Silvia Lopa

silvia.lopa@grupposandonato.it

Alessandra Colombini

alessandra.colombini@grupposandonato.it

Matteo Moretti

matteo.moretti@grupposandonato.it
ASCs Adipose-derived mesenchymal stem cell

BMAC Bone marrow concentrate

SVF Stromal vascular fraction

MRI Magnetic resonance imaging

VAS Visual analog scale
1 Cell and Tissue Engineering Laboratory, IRCCS Istituto Ortopedico Galeazzi, Via R. Galeazzi 4, 20161 Milan, Italy

2 Orthopaedic Biotechnology Lab, IRCCS Istituto Ortopedico Galeazzi, Via R. Galeazzi 4, 20161 Milan, Italy

3 Regenerative Medicine Technologies Lab, Ente Ospedaliero Cantonale, Via Tesserete 46, 6900 Lugano, Switzerland

4 Swiss Institute for Regenerative Medicine, Lugano, Switzerland 


$\begin{array}{ll}\text { WOMAC } & \begin{array}{l}\text { Western Ontario and McMaster Universities } \\ \text { osteoarthritis index }\end{array} \\ \text { HA } & \text { Hyaluronic acid } \\ \text { PRP } & \text { Platelet-rich plasma } \\ \text { ASA } & \text { Amniotic suspension allograft }\end{array}$

\section{Introduction}

Osteoarthritis (OA), the most widespread type of arthritis, is expected to become the fourth cause of disability by $2020[1,2]$, resulting in a relevant socioeconomic burden and affecting the gross domestic product of developed countries [3]. The establishment of adequate therapies able to counteract the progression of the disease and, hence, to prevent the loss of articular function and joint replacement is needed. In particular, the current conservative options, which include exercise and physiotherapy, and weight loss, with the use of analgesics and nutraceuticals, should be combined to yield more effective treatments. As symptoms escalate, anti-inflammatory drugs and intraarticular steroids can also be used to get pain relief and improve joint function [4]. However, in patients who do not respond to optimal conservative management, joint replacement represents the unique available therapeutic option. In this scenario, the development of efficacious conservative approaches would be particularly relevant to treat young individuals with early OA, since their more active and physically demanding lifestyle negatively correlates with the prosthetic implant survival [5].

\section{Overview on injective mesenchymal stem cell-based treatments}

Treatments involving the use of mesenchymal stem cells (MSCs), harvestable with minimally invasive procedures particularly from bone marrow and adipose tissue, are on the rise for the conservative treatment of OA [6]. MSCs have been demonstrated to be safe [7] and, in case of failure, they do not preclude any additional future treatment. Given the presence of diffuse chondral damages in osteoarthritic joints, the most common way to deliver MSCs in these patients is intra-articular injection. They have been used both in one-step procedure, as non-expanded cells, after in vitro expansion. The in vitro step allows for the selection of a more homogeneous cell population, meeting the standard criteria for MSC identification [8]. Furthermore, the number of cells administered to the patient can be precisely determined, ensuring a high reproducibility of the clinical procedure. On the other hand, therapies based on expanded cells involve a higher cost of the treatment. Additional concerns are related to the extensive in vitro cell manipulation, resulting in their classification as an advanced-therapy medicinal product (ATMP) and in the subsequent need to satisfy rigorous regulatory requirements for clinical use $[9,10]$. To overcome some of these limitations, it is possible to process by commercially available disposable devices both bone marrow and adipose tissue obtaining bone marrow aspirate concentrate (BMAC) and stromal vascular fraction (SVF) or micro/ nano-fragmented adipose, respectively. These products do not imply substantial cell manipulation and, thus, are not considered ATMPs. This makes their use easier not only from the technical point of view, since they are obtained in a single stage at the point of care, but also given the less complex regulatory pathway that has to be followed, although a clear position of the regulatory agents concerning the application of BMAC and SVF intra-articularly is still missing. However, the amount of MSCs present in these concentrates is usually lower compared to the doses of expanded MSCs that are administered to the patients, although this does not necessarily imply an inferior efficacy of the treatment [11]. Indeed, progenitor cell concentrates are a mixed cell population, including erythrocytes, leukocytes, and endothelial cells, and this allows maintaining MSCs in contact with their physiological cell niche, which is supposed to enhance their performances.

The features of the different MSC-based treatment pathways are illustrated in Fig. 1. Besides bone marrow and adipose tissue, other tissues have recently gained interest as a source of MSCs [6]. Considering an allogeneic use of these cells, non-expanded MSCs isolated from amniotic fluid and membrane have been used for the treatment of OA. Indeed, the use of allogeneic MSCs is possible given their low immunogenicity, which express low levels of MHC class I molecules and lack the expression of MHC class II and other co-stimulatory molecules. Furthermore, MSCs can inhibit the activity of several types of immune cells via cell-cell contact and paracrine signaling, which avoids immune responses in allogeneic recipients [12]. This approach offers some advantages in terms of clinical outcome over autologous MSCs in old patients or in patients affected by co-morbidities whose MSCs may have reduced regenerative and therapeutic potential [13-16]. Moreover, the use of potential off-the-shelf commercial preparations of allogeneic MSCs may reduce the overall cost of cell therapies, while maintaining an accurate quality control. Yet, it is reasonable to think that this approach might gain relevance in the treatment of OA once a more extensive characterization of the efficacy and safety of allogeneic MSCs will be available, as recently showed in two studies for the treatment of focal chondral lesions $[17,18]$. 


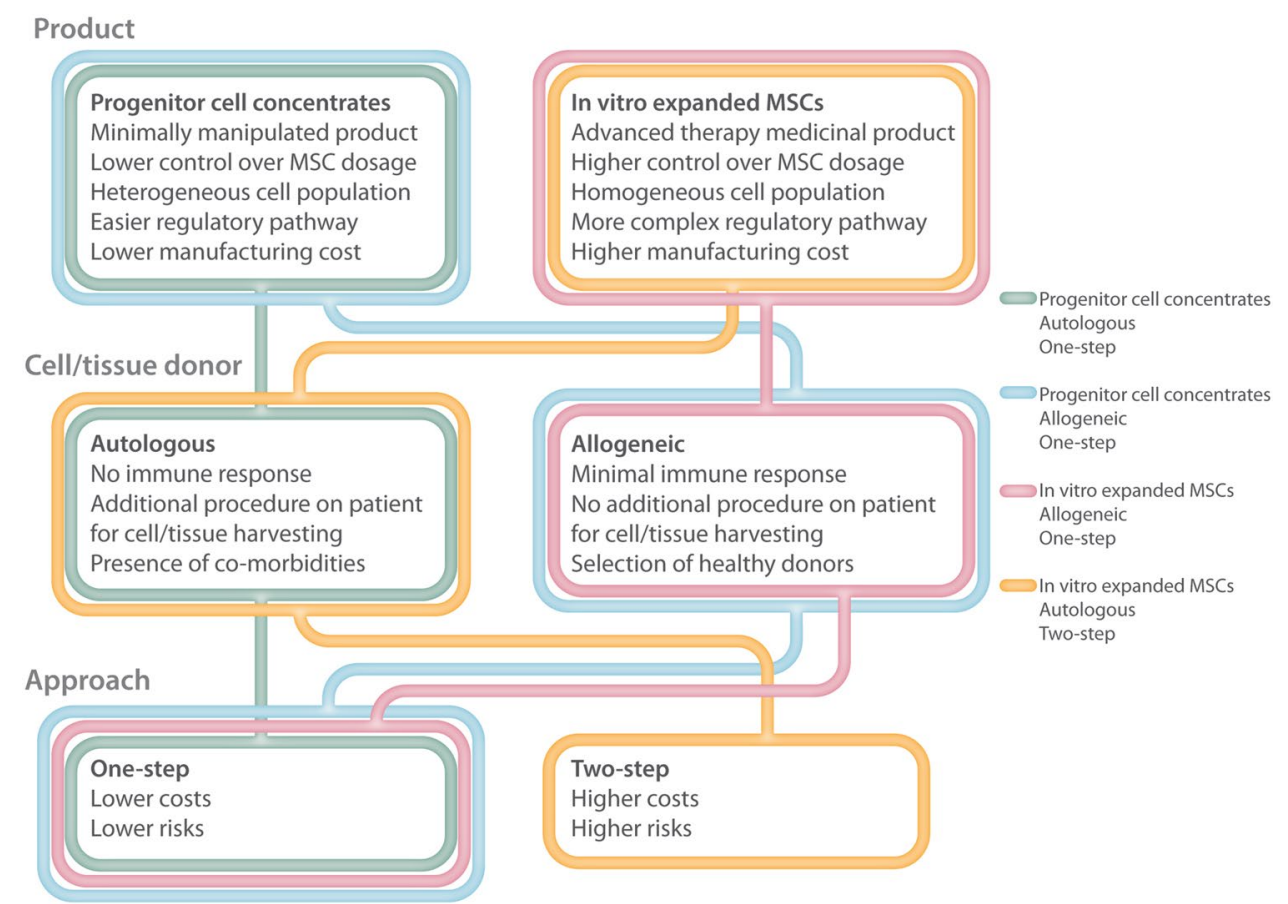

Fig. 1 Treatment pathways of MSC-based injective treatments for OA: features of products and donor sources. Progenitor cell concentrates can be considered as one-step approach. In fact, in the case of autologous progenitor cell concentrates (pink), cell/tissue harvesting and patient treatment are performed on the same moment. Allogeneic progenitor cell concentrates (blue), similar to allogeneic in vitro

\section{Aim of the review}

The purpose of this review paper is to provide the reader with the tools necessary to interpret the data, deriving from the available clinical studies concerning the intraarticular injection of MSCs, in the form of either expanded cells or progenitor cell concentrates, for the treatment of knee OA. We, indeed, believe that a good comprehension of the supposed mechanisms of action of MSCs might be very useful in the interpretation of the clinical effects of these treatments, as well in the critical analysis of the quality of the studies presented here. Reviewing the existing literature, we selectively focused on injective conservative procedures to evaluate the effects of the paracrine activity of MSCs in a pathological joint, aiming to answer the question: "Do the current results about the use of MSCs-either as freshly harvested or after culture expansion-for the treatment of OA support the supposed mechanisms of action of these cells?". This analysis includes the papers, other than case reports, presenting patients affected by Kellgren-Lawrence (KL) grades I-IV, in which the injective treatment was never associated with surgical procedures of the affected knee(s). In this way, we tried to avoid confounding factors that may have made data expanded MSCs (green), are "off-the-shelf" products, compatible with a one-step intervention on the patient. The use of autologous expanded MSCs (yellow) is the only treatment that involves a twostep approach, since the patient undergoes cell/tissue harvesting and treatment in two separated moments. Professional illustration by Matilde Bongio, Ph.D., GoArts-IRCCS Istituto Ortopedico Galeazzi

interpretation even trickier. Tables $1,2,3,4$ provide the reader with an easy-to-consult summary of all the studies currently published in the literature following the aforementioned characteristics. Detailed information regarding the characteristics of the patient cohort, the study design, the assessments, and the main outcomes of each study is reported, while only the most relevant publications are commented in the following paragraphs.

\section{Why should MSCs work in OA?}

The convenient but debated term "MSCs" has been used to describe virtually any ex vivo expanded stromal cell population. For more than 3 decades, the rationale for the use of MSCs in musculoskeletal applications has been their ability to differentiate into tissue-specific cell types, such as osteoblast-, chondrocyte-, and tenocyte-like cells. A dramatic cultural revolution started about 10 years ago when the scientists consistently focused their attention on the ability of these cells to "sense" the environment and secrete as a response large quantities of different bioactive molecules, such as cytokines, antioxidant and pro-angiogenic substances, trophic factors, and other proteins [45]. In 


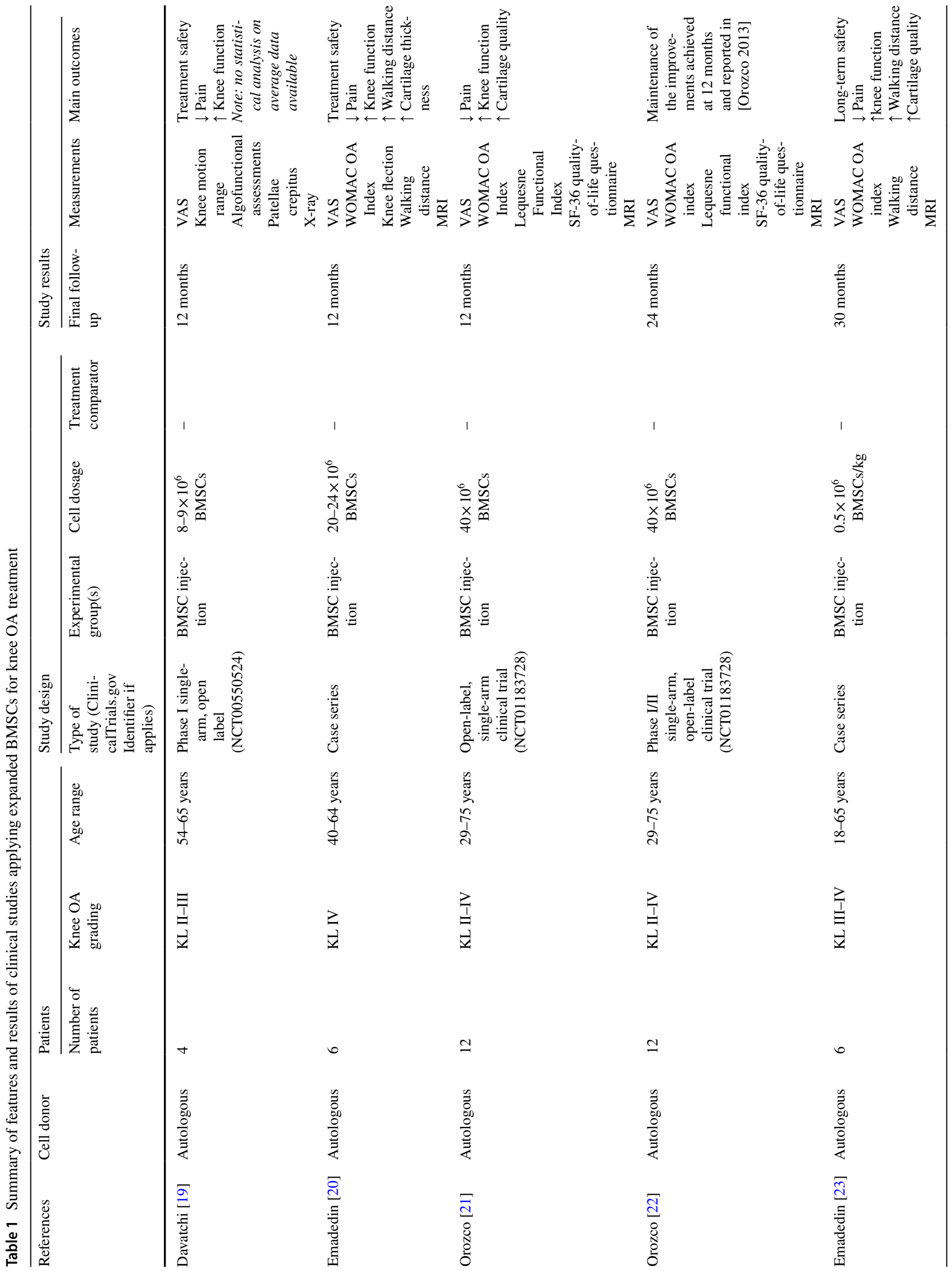




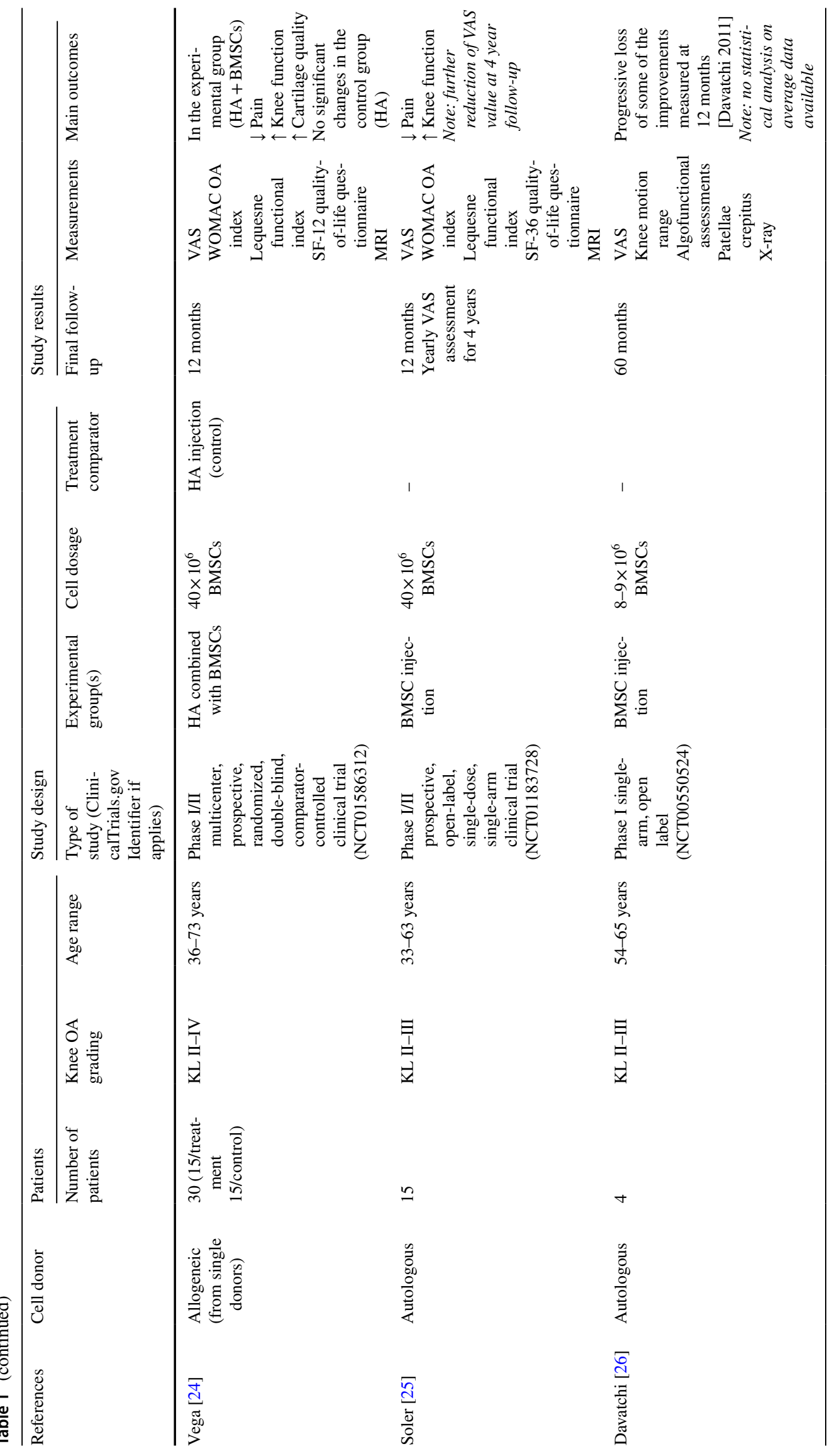




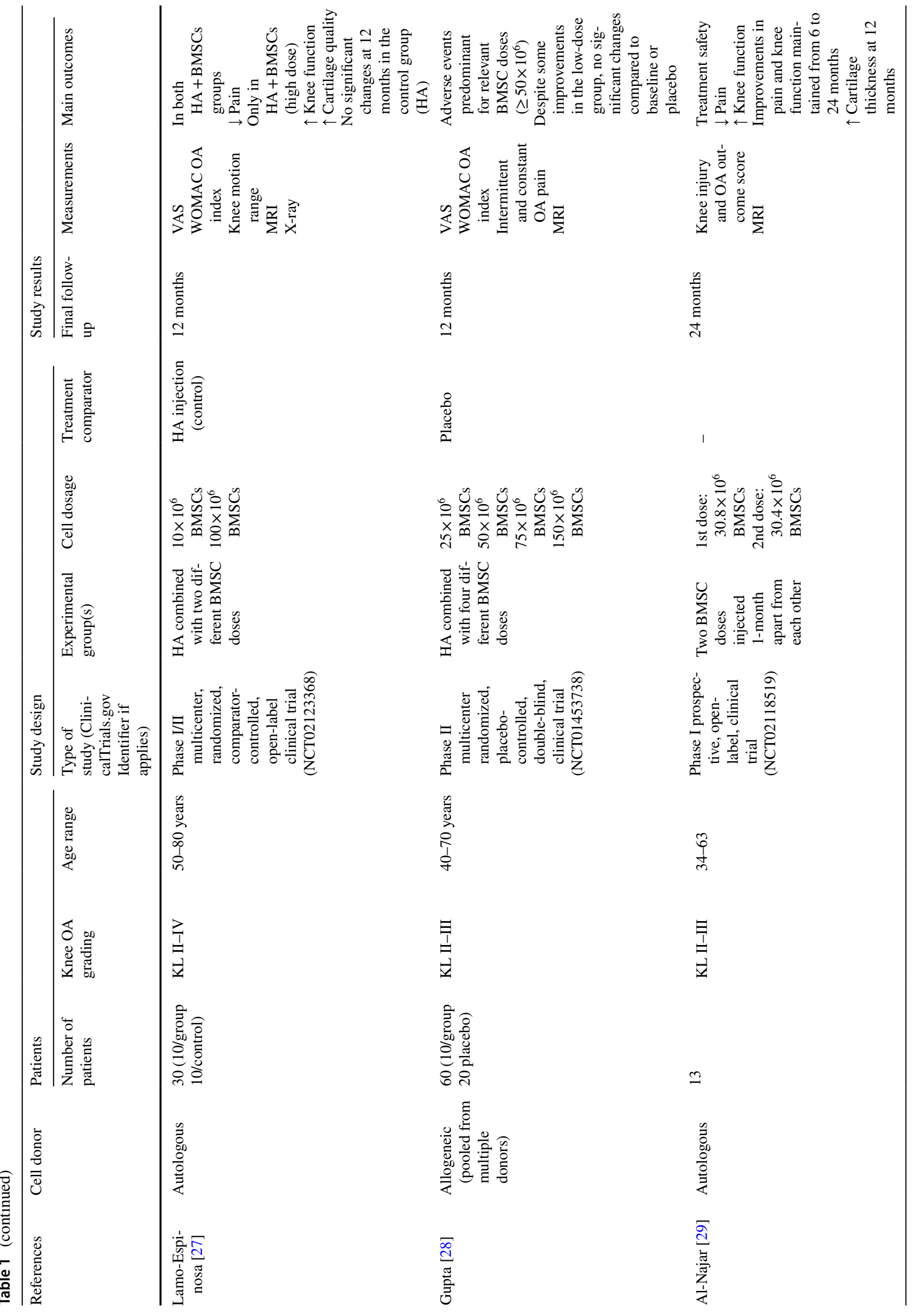




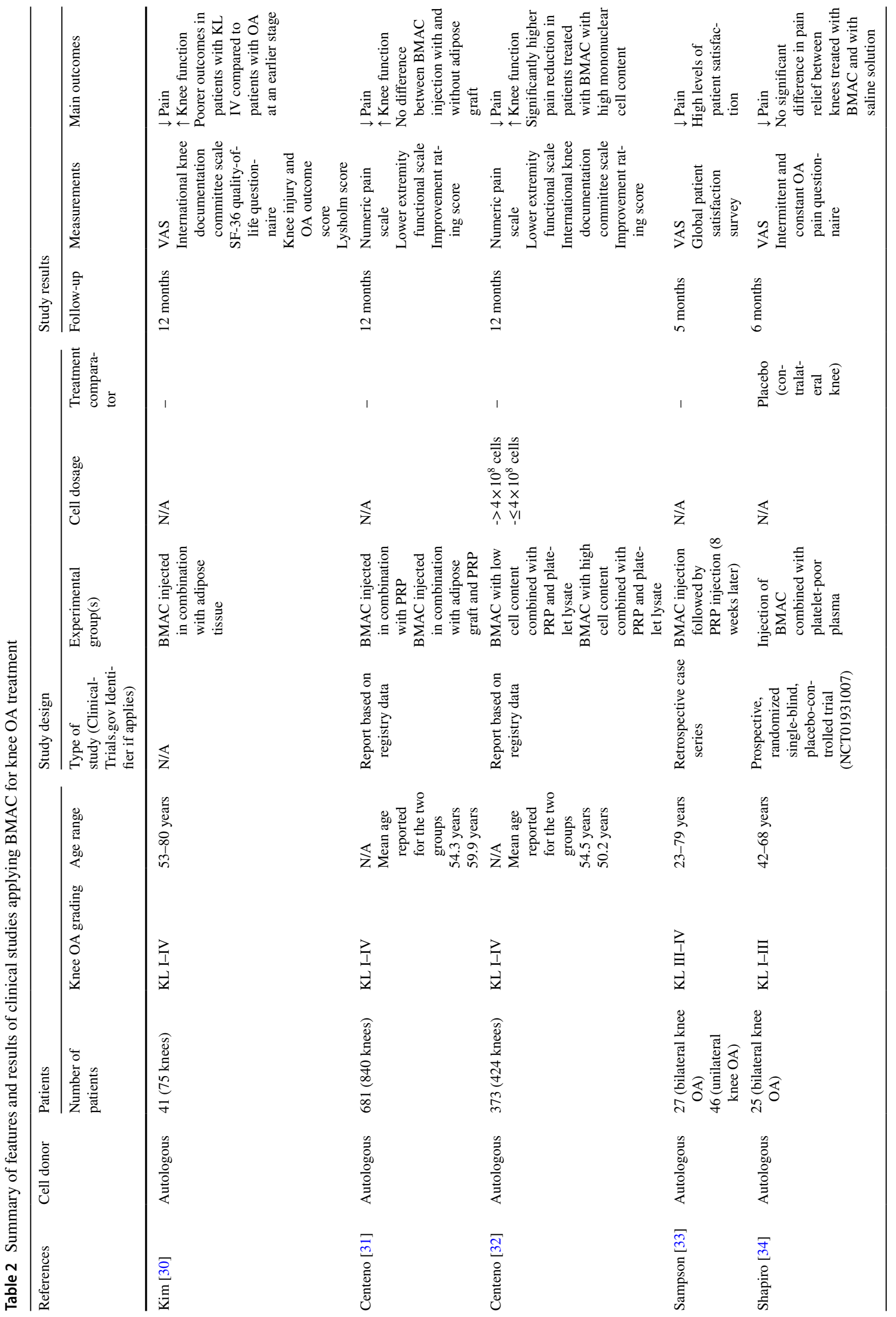




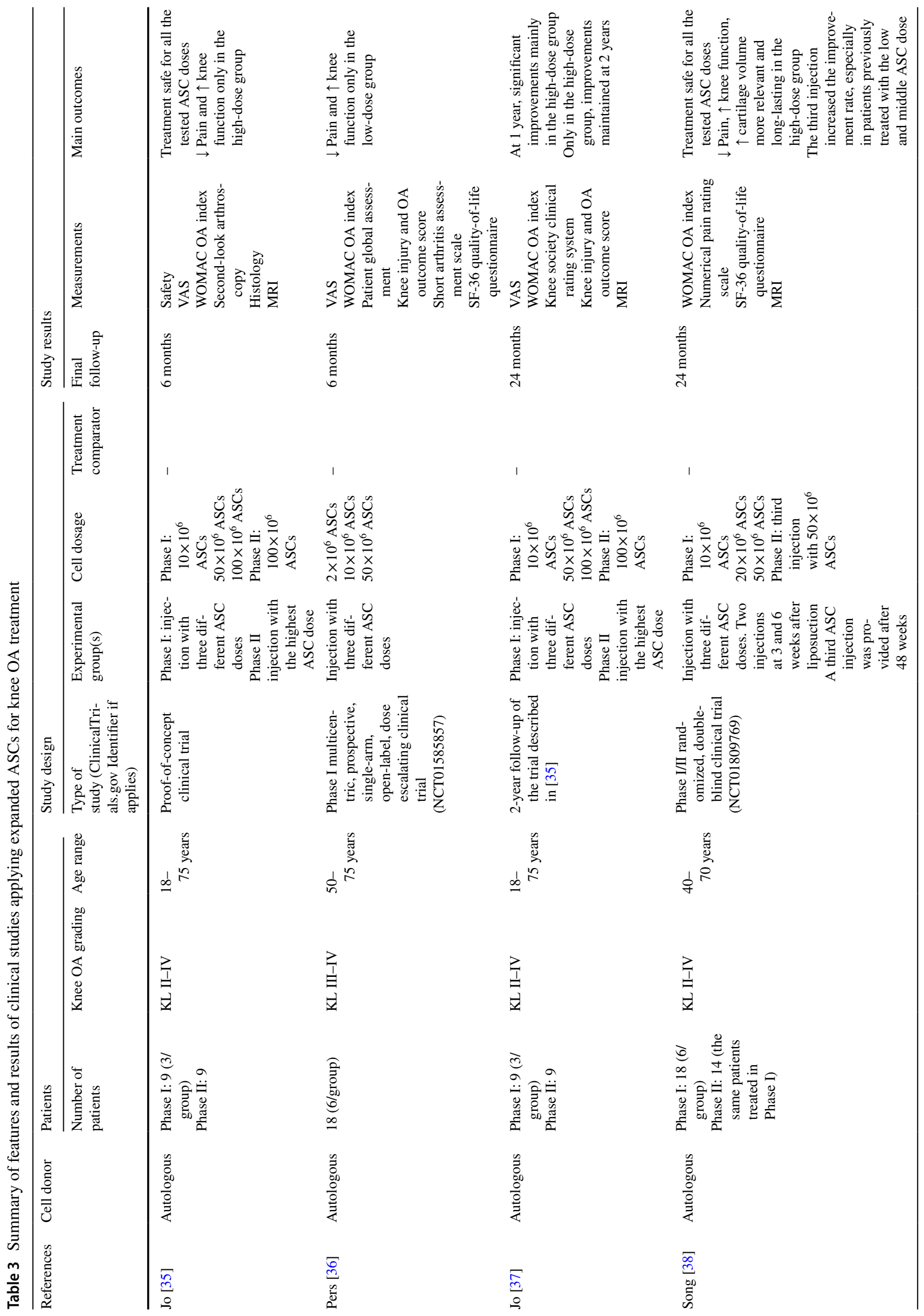




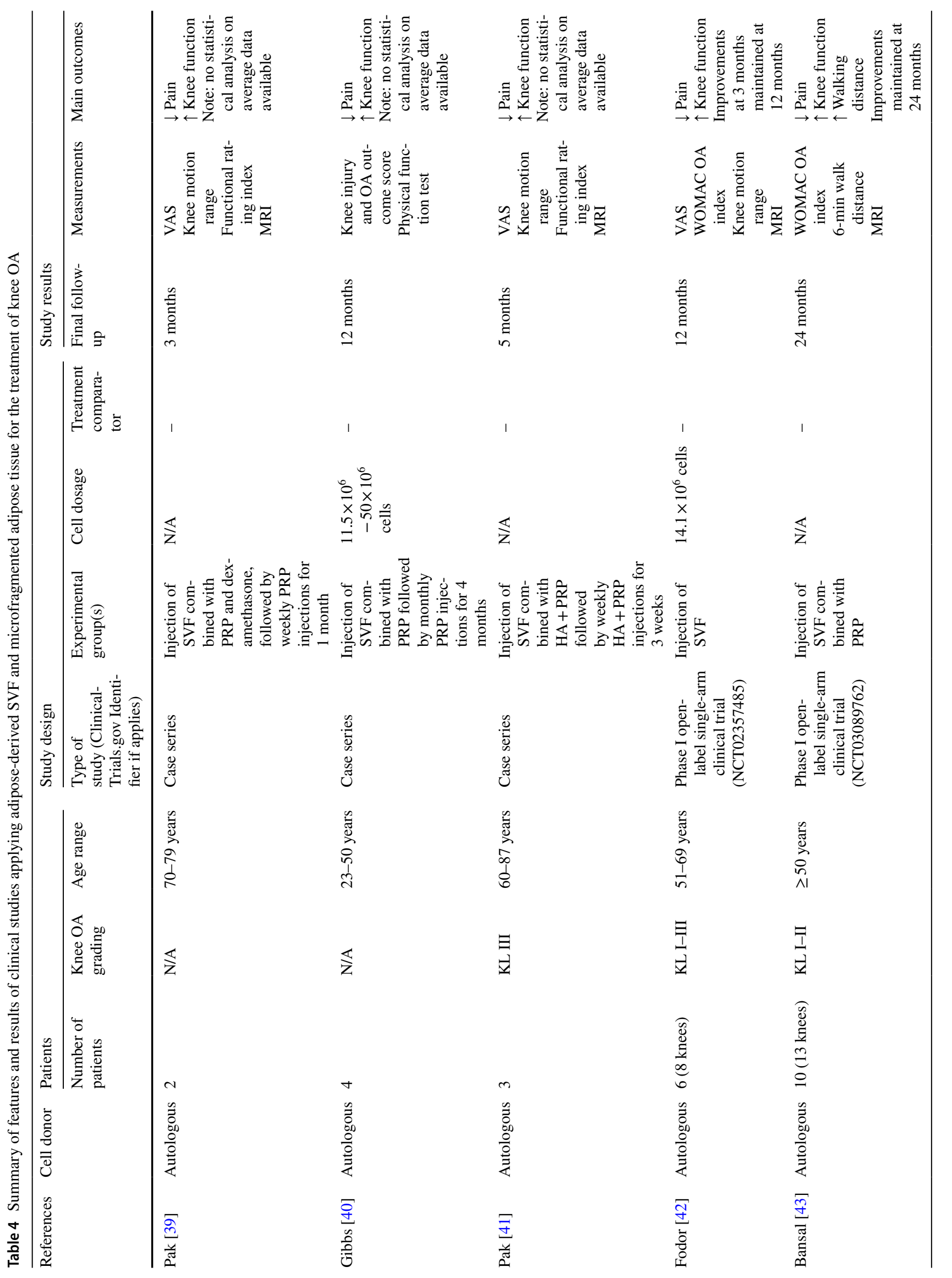




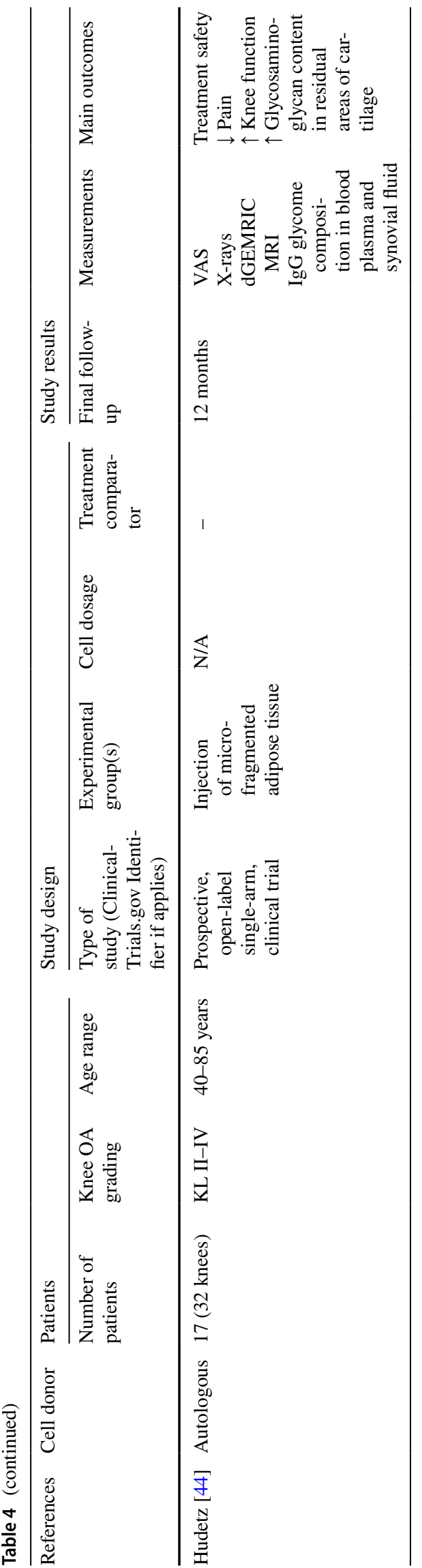

physiological conditions, MSCs reside in the perivascular niche in a quiescent condition until the signals released after an injury activate their migration to the damaged site promoting the production of bioactive molecules to re-establish tissue homeostasis [46-48]. For this reason, they have been recently renamed as "medicinal signaling cells" [46, 47, 49]. As a result, the paracrine activity of MSCs would be involved in productive repair, by switching off inflammation, limiting stress response, and apoptosis, and recruiting the immune and reparative cells of the recipient [50-53]. An extensive in vitro and ex vivo research activity focused on the identification and explanation of the mechanisms of action of MSCs. Some of these studies clearly report an influence of MSC paracrine activity on inflammation and matrix turnover in OA, where the presence of a pro-inflammatory milieu was suggested as the switcher to promote the anti-inflammatory effects of MSCs. Indeed, priming BMSCs with OA synovial fluid promotes an increase of indoleamine 2,3-dioxygenase (IDO) expression [54], while priming these cells with IFN $\gamma$ and TNF $\alpha$ determines an increase of IDO activity and IL-6 expression [55]. Moreover, conditioned medium obtained from BMSCs primed with OA synovial fluid inhibits T-cell proliferation [54], while, after IFN $\gamma$ and $\mathrm{TNF} \alpha$ priming, BMSC-conditioned medium determines IL-1 $\beta$ downregulation and SOCS1 (suppressor of cytokine signaling) upregulation in synovium explants, and a downregulation of ADAMTS5 and upregulation of IL-1Ra and SOCS1 in cartilage explants [55]. In addition, in the presence of high levels of pro-inflammatory mediators, a coculture in transwell of ASCs obtained from infrapatellar fat pad, subcutaneous hip, or abdominal fat with chondrocytes and synoviocytes determines a decrease of IL- $1 \beta$, IL- 6 , and CXCL8/IL-8 expression and release [56]. Finally, amniotic stem cells in co-culture with explants of cartilage and synovium have been shown to improve chondrocyte viability and cartilage glycosaminoglycan content as well as to provoke a shift of synovial macrophages towards an antiinflammatory phenotype [57].

All these recent observations do not invalidate the "old school" theory based on the participation of MSCs to the repair process through their direct differentiation into tissuespecific cells. However, it is hard to believe that the few MSCs contained in a BMAC or SVF preparation injected intra-articularly can reach the multiple chondral lesions, permanently adhere and start the repair process by producing new cartilage. Therefore, while these two mechanisms of action of MSCs (paracrine action and direct cell differentiation) are not exclusive of each other, the difference is essential and needs to be taken into account for a critical analysis of the literature and for informing correctly the patients about the reasonable results they should expect from this kind of treatment. 


\section{Clinical outcomes of MSCs in the treatment of $O A$}

\section{Bone marrow-derived products}

\section{Bone marrow-derived MSCs (BMSCs)}

Autologous expanded BMSCs have been the election choice in the majority of the clinical studies reported so far, although, on two small cohort of patients $(n=6)$, two studies of the same authors $[20,23]$ showed that the treatment with expanded BMSCs $\left(20-24 \times 10^{6}\right.$ cells and $5 \times 10^{5} \mathrm{MSCs} /$ $\mathrm{kg}$, respectively) allowed for an increase in cartilage thickness and extension of the repair tissue over the subchondral bone, as well as a significant improvement in Western Ontario and McMaster Universities OA Index (WOMAC) up to 30 months. These improvements remained stable up to 12 months with a following decrease, thus suggesting that subsequent injections of MSCs may be needed to achieve prolonged therapeutic efficacy. On the other hand, other studies have reported more durable outcomes of the intra-articular delivery of similar single doses of autologous expanded BMSCs. The injection of $40 \times 10^{6}$ BMSCs in 12 patients with KL grades II-IV allowed for significant improvements over time of pain functional scores (VAS) and articular cartilage quality, without any decrease between $12-$ [21] and 24-month follow-up [22]. Another group reported the results up to 5 years $[19,26]$ post $8-9 \times 10^{6}$ autologous BMSC injection on four patients with bilateral middle or advanced knee OA. Although a progressive deterioration was observed, at the last follow-up, the outcomes were still better than the baseline, thus suggesting a protective role of MSCs, since the untreated knee continued its progression towards degeneration. However, despite the advantage of having results at a very long follow-up for the same patients, due to the very limited number of patients and the lack of mean values and statistical analysis, it is impossible to draw any robust conclusion about the length of the therapeutic efficacy of the described procedure. A very recent study [29] has reported significant and stable improvements in terms of pain, knee function, and quality of life up to 24 months after two subsequent injections, with an interval of 1 month, of about $30 \times 10^{6}$ BMSCs. Given the absence of contraindications in repeating this treatment, repeated doses of cells might be a solution to prolong the effectiveness of the results.

As can be inferred by the aforementioned studies and the others reported in Table 1, there is not a consensus about the ideal therapeutic dose for intra-articular treatment of OA. In the attempt to clarify this point, a phase I/II multicenter randomized-controlled trial at 12-month follow-up tested different doses of autologous expanded BMSCs $\left(10 \times 10^{6}\right.$ and $100 \times 10^{6}$ ) in association with hyaluronic acid (HA) in 30 patients with knee OA. Patients treated only with HA represented the control group [27]. Both doses of BMSC allowed for a significant VAS improvement with respect to baseline, where improvement in WOMAC was reported only for the patients treated with the highest dose. HA alone failed to improve symptoms at 12 months. Moreover, only the administration of the high dose of BMSCs halted the progressive loss of articular cartilage, indicating that a low dose of BMSCs may not be sufficient to obtain stable functional improvements and to significantly impact tissue quality.

The use of allogeneic BMSCs represents an alternative to autologous cell-based therapies. A recent randomizedcontrolled trial [24] showed significantly better results in 15 patients KL II-IV treated with intra-articular injection of $40 \times 10^{6}$ allogeneic BMSCs in terms of VAS, WOMAC, Lequesne indices, and articular cartilage quality compared to the control group injected with HA only at 12-month follow-up. In a randomized double-blind multicentric placebo-controlled phase II study [28], four different doses of allogeneic BMSCs pooled from multiple donors (Stempeucel®) were tested on 10 patients KL II-III each, for a total of 40 patients, whereas the remaining 20 patients received a placebo injection. The lowest doses of BMSCs $\left(25 \times 10^{6}\right.$ and $50 \times 10^{6}$ cells) were safe and tolerated, while the highest dose groups $\left(75 \times 10^{6}\right.$ and $150 \times 10^{6}$ cells $)$ yielded adverse events, mostly knee pain and swelling. Despite some positive trends in the $25 \times 10^{6}$ group, none of the clinical parameters was significantly improved and no relevant changes in X-ray and MRI were observed compared to baseline. This indicates once again that the clinical efficacy of such therapies should be verified on a large patient cohort to achieve consistent results. Moreover, although MSCs are considered poorly immunogenic, still, they can elicit an immune response when used in allogeneic way, as shown by these results, and thus, the identification of a correct dose is even more crucial in this context.

\section{Bone marrow aspirate concentrate (BMAC)}

There are several commercial systems that allow clinicians to quickly recover concentrated, patient-derived nucleated cells, platelets, and other soluble factors in the form of BMAC. Most of these automated systems are based on gradient separation by centrifugation in a semi-closed or closed apparatus, and allow to achieve volume reduction and a 2-8X total nucleated cells with respect to the unprocessed bone marrow.

A recent single-blind placebo-controlled trial including 25 patients [34] showed a significant pain reduction after 6 months, but without difference with respect to placebotreated contralateral knee. This lack of difference raises 
some concerns about the extent of the placebo effect when patients are included in a trial, but, at the same time, it raises several interpretative doubts; in fact, the pain relief at the contralateral (control) knee may have been affected by the reduction of symptoms on the target one, and also, since the same patient represented both the treatment and the control group, this may have led to a tricky subjective evaluation. Certainly, an objective evaluation of the patients might have helped to give a more accurate interpretation of the findings of this study. A retrospective case series including 73 patients with knee OA [33] with a 5-month follow-up showed that intra-articular injection of BMAC followed by PRP injection after 8 weeks resulted in a significant pain reduction and high patient satisfaction. However, cartilage quality was not assessed, and the combination of BMAC and the subsequent treatment with PRP does not allow distinguishing the therapeutic effect of BMAC alone. The data at 12-month follow-up of a registry including 373 (424 OA knees) patients that received BMAC injections for the treatment of OA showed significant improvements for all the reported pain and functional parameters compared to the baseline [32]. The authors set a threshold of $4 \times 10^{8}$ cells to divide the patients in groups receiving a low dose and a high dose of total mononuclear cells, demonstrating more benefits in the high-dose group. This result seems to indicate that the number of progenitor cells, even when used without any cell expansion, could affect the outcomes. For this reason, the collection of data regarding mononuclear cell count in BMAC and an improvement in the standardization of cell counting would contribute to generate comparable data regarding the efficacy of BMAC injective treatments. It needs to be highlighted, indeed, that given the intraoperative setting of the use of BMAC, most of the studies about this approach do not provide any information about cell dose.

Besides cell content, also the stage of the disease appears to be a determinant in the outcome of these therapies. In a study conducted on 41 patients (75 knees) treated with BMAC injections in combination with adipose tissue used as a sort of scaffold to deliver more efficiently BMAC, the pain and functional scores improved in all the patients. Interestingly, the treatment yielded poorer results in patients with late-stage OA (KL IV) than in patients with the early/middle-stage OA (KL I-III) [30]. The combination of BMAC with adipose tissue was also analyzed in a registry reporting data of patients who underwent BMAC procedures with (224 procedures) and without (616 procedures) an adipose graft. While the pre- and post-treatment improvements were statistically significant in both groups, the differences between the groups were not, suggesting that addition of an adipose graft to BMAC did not provide any relevant benefit [31]. Again, this study only provides data relative to subjective algofunctional assessments, while it would be interesting to investigate if the addition of the adipose graft provides any improvement in articular cartilage quality to evaluate the risk-benefit ratio of performing an additional liposuction procedure.

\section{Adipose tissue-derived products}

\section{Adipose tissue-derived MSCs (ASCs)}

The safety of high stem cell dosages for intra-articular injection has been investigated also in the context of adipose stem cell-based therapies. After having assessed the safety of three different autologous expanded ASCs' doses $\left(10 \times 10^{6}, 50 \times 10^{6}\right.$, and $100 \times 10^{6}$ cells $)$ injected intra-articularly in patients affected by knee OA [35], a phase II study including nine additional patients treated with the highest cell dose was carried on. The results showed significantly better clinical results in the high-dose group with respect to the lower ones, suggesting that an adequate number of MSCs are crucial to achieve relevant clinical benefits. This result was further confirmed by a subsequent follow-up study, which reported that significant improvements at 2 years were maintained only in the high-dose group [37]. Completely opposite results were reported by another recent study [36] that tested different doses of autologous ASCs in a phase I clinical trial including 18 patients with symptomatic and severe knee OA. Of the three different ASC doses $\left(2 \times 10^{6}\right.$, $10 \times 10^{6}$, and $50 \times 10^{6}$ cells), while all showed a satisfactory safety profile, significant improvements in terms of pain, function, and mobility were observed only in patients treated with the lowest dose of ASCs at 6-month follow-up. The apparent conflicting result of these studies $[35,36]$ may have been affected by a common bias, since the patients who exhibited the best response to ASC treatment had the worse baseline scores. Certainly, this may be ascribed to the lower expectations and a better predisposition to perceive any post-therapy improvements in these patients. However, these results can also be read as the need of an inflamed milieu to prime the injected ASCs and make them exert their homeostatic function at best, as demonstrated by ex vivo experiments. Once again, the heterogeneity and the limited number of patients included in the clinical trials preclude the possibility of a straightforward result interpretation, indicating that prospective trials on larger patients cohorts with a careful randomization based on the disease stage are needed. As already reported for BMSCs, repeated injections of $50 \times 10^{6}$ ASCs allowed for significant improvements in terms of pain and knee function. An additional injection provided at 48 weeks after the previous ones was able to generate another improvement, positively affecting also the cartilage volume [38]. 


\section{Stromal vascular fraction (SVF) and micro/ nano-fragmented adipose tissue}

A number of different systems to recover the "regenerative component" of adipose tissue have been recently introduced into the market. To comply with the rules of minimal manipulation, the tissue processing must avoid the use of enzymes or other molecules, and thus, generally, the digestion is mechanical. The isolation of SVF first requires a digestion of the extracellular matrix, usually followed by a centrifugation phase where SVF cells are concentrated. An alternative to the isolation of SVF is represented by the so-called micro- or nano-fragmented adipose tissue, belonging to the family of fat transfer. In this case, the extracellular matrix of the tissue is not removed, maintaining the tissue microarchitecture and an intact stem cell niche [58, 59].

Regarding the injection of SVF for the treatment of knee $\mathrm{OA}$, a few studies showed improvements in the clinical and functional outcomes [39-41] up to 2-year follow-up, in some cases, also associated with radiological improvements. However, the very poor number of patients included in these studies and the concomitant use of other therapeutic agents like PRP, dexamethasone, or cavitation, do not allow for a clear identification of the effect of SVF. More recently, a study involving ten knee OA patients treated with SVF and PRP showed a reduction of pain, a functional improvement at 2 years of follow-up, and an increase of cartilage thickness after 1 year in six out of ten patients [43]. However, the quality of cartilage remains one of the main elements of discussion, given that, so far, just in a few cases, a stable hyaline cartilage was found after cartilage procedures. Therefore, it would be more appropriate to refer to cartilage repair. Positive effects have been reported also when using SVF alone. These include a functional improvement at 3 months and pain relief after 1 year shown in a study including 6 patients with knee OA [42]. Finally, a very recent study has reported the use of micro-fragmented adipose tissue in 17 patients (32 treated knees) showing significant improvements in terms of pain and cartilage quality up to 12 months [44]. Interestingly, this study used the dGEMRIC (delayed gadoliniumenhanced MRI of cartilage) protocol for the MRI assessment to determine changes pre- and post-treatment in glycosaminoglycan content in specific cartilage regions, as a measure of the trophic and paracrine actions of progenitor cells on resident chondrocytes, which yields more specific information than cartilage thickness assessed with standard MRI. Despite these encouraging evidences, the relatively low number of patients enrolled in these studies does not allow withdrawing definitive conclusions about treatment efficacy and further studies on larger patient cohorts are required to select the best strategy/device to use and demonstrate the long-term efficacy of this approach.

\section{Alternative source of MSCs}

\section{Amniotic fluid cells}

Amniotic suspension allografts (ASAs), containing particulate human amnion and amniotic fluid cells, have also been proposed for the treatment of symptomatic knee OA [60]. In an open-label prospective study on six patients with knee $\mathrm{OA}$, it was demonstrated that a single intra-articular ASA injection from allogeneic donors allowed for significant improvements in pain and functional scales observed up to 12 months. These results indicated this novel cell source as an alternative tool for OA treatment, paving the way to a larger, placebo-controlled randomized trial to further assess treatment efficacy that recently completed patients' enrolment.

\section{The lesson learned}

\section{What we should expect from OA patients treated with MSCs?}

A not negligible number of studies assessing the efficacy of MSC, either freshly harvested or culture-expanded, for the treatment of OA have been published so far. This demonstrates the interest of the scientific community for this conservative approach that may potentially change the treatment scenario of this very common and disabling disease. Considering the in vitro and in vivo findings reported so far, when treating an OA joint, we should expect a resolution of symptoms, at least transient, given the ability of MSCs to sense the environment and secrete, accordingly, a plethora of trophic and immunomodulatory molecules. This has been, indeed, reported in most of the studies analyzed here, although with different extent and durability. Durability of this treatment and more in general of all the "biological" treatments included PRP is one of the main points of discussion. The detractors of MSC-based treatments criticize the duration of the effects, that in some cases was less than a year, whereas in other cases lasted up to 2 years [22, 37,43 , with preliminary evidences of even a longer effect although progressively decreasing with time [26]. However, it should not be surprising that these treatments cannot lead to a definitive resolution of the disease. Indeed, injecting these therapeutic agents locally, it is just possible to modulate the microenvironment the cells found once delivered in the joint, without being able to counteract the inexorable progression of OA. MSCs, differently from PRP that has a short in vivo half-life [61], can survive longer in a joint cavity and keep releasing molecules. However, it is hard to predict the length of MSCs life once delivered, given the hostile microenvironment which they encounter, which is 
Fig. 2 Synovial, cartilaginous, and bony-derived markers of degradation, synthesis, and inflammation in a joint affected by OA [64]. The paracrine activity of the MSCs in the OA articular environment resides in their anti-inflammatory, anti-catabolic, and trophic abilities. Monitoring of variations in these markers has been proposed as a strategy to evaluate the efficacy of MSC-based OA treatments. Professional illustration by Matilde Bongio, Ph.D., GoArts - IRCCS Istituto Ortopedico Galeazzi

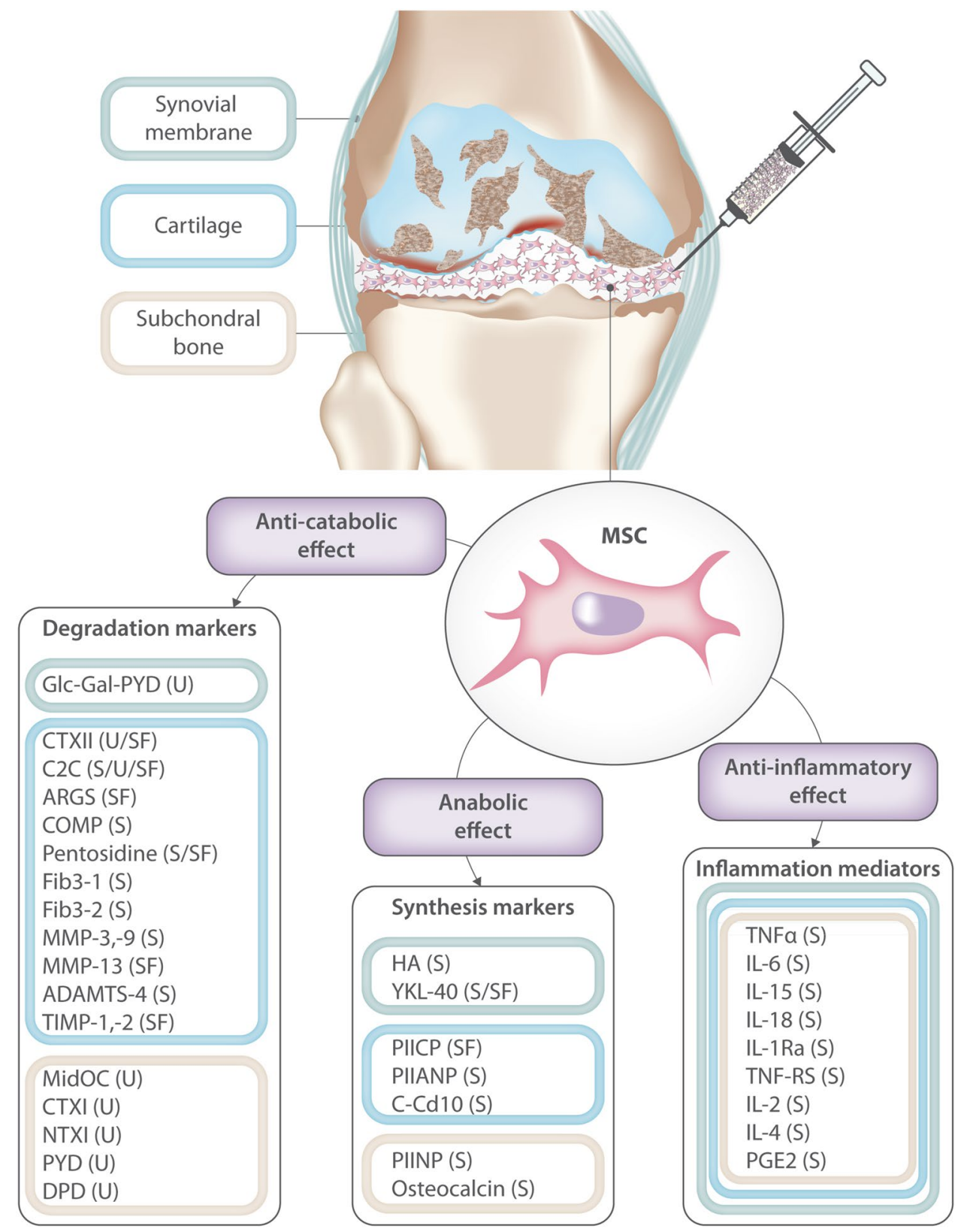

of times, the use of repeated cell injections upon symptoms which return appears a reasonable approach to take full advantage of this technique.

It is well known that there is a high subjectivity of the patient response to the conventional synthetic drugs, and this is strongly dependent on the patient's characteristics, since the drug formulation is consistent and standardized. When receiving a MSC treatment, the grade of complexity in predicting the patient's response dramatically increases given the lack of standardization of the MSC preparation, especially those prepared at the point of care, which may affect the features of the final product. This does not mean that MSCs do not have therapeutic effects. Indeed, in our opinion, the lack of result homogeneity highlighted by 
some review papers [63] may be expected and not necessarily intended as a negative finding. For this reason, we do not agree with the authors that expect to find unambiguous proof of MSC efficacy comparing different types of studies involving dramatically different patient types and sometimes comparing the therapeutic outcome in different joints. Rather, we believe in a correct interpretation of the findings of the single studies that may lead to important conclusions if they are well designed. Moreover, likewise PRP, the quality and properties of BMC and SVF/micro-fragmented adipose tissue are strongly affected by the method of preparation and device used. For this reason, once again, it would be methodologically uncorrected to pool the results of different techniques, which, instead, need to be analyzed separately.

While the medical community well accepts the idea of chronic pharmacological protocols to give a stable relief from the target disease, it seems harder to accept the idea that the effects of a one-shot cell-based treatment cannot last forever. What we should have learned so far is that we cannot aspire to compare cell-based products, above all freshly prepared cell concentrates, with the conventional drugs, given the aforementioned substantial differences. At the same time, interpreting the results of cell-based treatments cannot even be compared to surgical treatments such as joint replacement, that, of course, provide much more durable results but imply an incomparable invasiveness and possible side effects which many patients are not ready or not yet in the need to face.

\section{How could we improve the knowledge about MSCs' treatment for knee OA?}

While we acknowledge that the inconsistency of the results reported in the literature is affected by the intrinsic characteristics of this treatment, at the same time, we claim for a more rigorous approach in conducting studies. Among all the studies reported (Tables 1, 2, 3, 4), only four of them (14\%) present a control group such as HA or placebo. Even worse, more than one-third (37\%) of the studies show the results on less than ten patients, and thus, considering the high inter-patient variability hardly allows for any deduction. More controlled trials as well as multicentric studies allowing to recruit higher number of patients are needed, especially for adipose-derived MSC treatments, which have been more recently introduced with respect to bone marrow ones. The design of these studies is crucial and deserves much attention to achieve consistent and easy-to-interpret outcomes. The satisfactory, somehow striking results, obtained by scientists in vitro and in vivo encouraged the rapid translation of MSC-based therapies. However, while it is easy to provide an optimal in vitro setting for MSCs to grow and perform, as well as to analyze the objective results in animal models (histological/biochemical analysis), the clinical setting is far from offering these possibilities, and thus, translating these approaches into successful clinical protocols has proven to be trickier than probably expected. This line of investigation is particularly challenging in the absence of tools that allow the identification of objective improvements following MSC treatments. Hence, the scientific community urgently needs to define a panel of standard outcome measures and suitable time points to evaluate the efficacy of the therapy. Specific MRI protocols, such as dGEMRIC, have been proven to be more sensitive and give more conclusive information about the actual quality of the repaired cartilage, instead of only focusing on cartilage thickness, which, not surprisingly, may not be affected by this treatment, especially in older patients, or affected by severe OA. Rather, new and more specific tools should be used to evaluate the possible modulation of the local environment after a MSC treatment. Amongst them, biomarkers that can be assessed in body fluids, such as blood plasma, urine, and synovial fluid (Fig. 2), represent an invaluable and non-invasive tool to monitor over time the efficacy of MSCbased treatments. Monitoring the variations in biomarkers will allow to specifically focus on the trophic and/or immunomodulatory activity of MSCs, thus shading further light on the in vivo mechanisms of action of these biological therapeutics and correlating them with the observed clinical improvements. To note that accurate longitudinal studies might be very useful too, as they would provide data on a large variety of patients, which later can be stratified and analyzed. In this view, the creation of common registries shared by a group of centers and including relevant patients data and information that would allow for a critical outcome interpretation seems to be a smart option to further improve the knowledge in this field.

\section{Conclusions}

Although substantial data have been published to date mostly accompanied by satisfactory results, the complexity of MSC metabolism and related therapeutic effects does not allow withdrawing definitive conclusions about the superiority of one tissue source over another, as well as about the best cell dose (if measurable) and the long-term durability of the effects of these procedures. Despite this, we are convinced that MSCs will have an important role in the conservative treatment of $\mathrm{OA}$ and that the research needs to continue to improve our knowledge. From a practical point of view, although less explored, one-step procedure, implying the use of autologous unexpanded sources of MSCs, presents advantages that cannot be questioned in comparison with two-step approaches. Much work needs to be done to carefully define the clinical circumstances of OA joints, but even more the characteristics of patients where these techniques should be 
utilized as well as their in vivo mechanisms of action. This should be achieved using adjunctive innovative technologies, such as cellular and molecular approaches, to define a complete picture of the patients in terms of local and/or systemic levels of inflammatory and metabolic markers of pathology. A more accurate monitoring of the complexity of the OA biology will further help in the early diagnosis and in the evaluation of its evolution. To accomplish this task, a strict collaboration between basic scientists, clinicians, industry, and regulatory agencies is needed to gain a better understanding of the very complex phenomena behind MSC therapeutic effects.

With a look towards the next future, the assumption that the therapeutic effect of MSCs in the treatment of OA relies on their secreting response to the local microenvironment signals clears the way to isolate the MSC-derived "healing" factors, the so-called secretome. The use of secretome would mean to take advantage of the product secreted by the cells without the use of the whole living cells, allowing to avoid the risks and discomfort of cellular transplantation. While the secretome will permit overcoming some of the limitations of cell-based therapies, it will deserve even more attention before being implemented in clinical setting given the need of an in vitro preparation and standardization. The current insights should help the scientific community to design more informative and revealing experiments that will lead to a more accurate translation into practical and effective clinical treatments. This field of investigation is very active and the promising preliminary evidences have been already observed in animal models of different diseases, confirming the feasibility of the approach that may demolish further barriers in the use of MSC-derived products.

Funding The salary of the authors was partially funded by the Italian Ministry of Health (Project Line 1, Progressive ID 28; Project Line 4 Progressive ID 98).

\section{Compliance with ethical standards}

Conflict of interest Laura de Girolamo is scientific consultant for Lipogems International S.p.A. The other authors declare that they do not have conflict of interests.

Open Access This article is distributed under the terms of the Creative Commons Attribution 4.0 International License (http://creativeco mmons.org/licenses/by/4.0/), which permits unrestricted use, distribution, and reproduction in any medium, provided you give appropriate credit to the original author(s) and the source, provide a link to the Creative Commons license, and indicate if changes were made.

\section{References}

1. Glyn-Jones S, Palmer AJ, Agricola R, Price AJ, Vincent TL, Weinans $\mathrm{H}$ et al (2015) Osteoarthritis. Lancet 386:376-387
2. Woolf AD, Pfleger B (2003) Burden of major musculoskeletal conditions. Bull World Health Organ 81:646-656

3. Hiligsmann M, Cooper C, Arden N, Boers M, Branco JC, Luisa Brandi $\mathrm{M}$ et al (2013) Health economics in the field of osteoarthritis: an expert's consensus paper from the European Society for Clinical and Economic Aspects of Osteoporosis and Osteoarthritis (ESCEO). Semin Arthritis Rheum 43:303-313

4. Anandacoomarasamy A, March L (2010) Current evidence for osteoarthritis treatments. Ther Adv Musculoskelet Dis 2:17-28

5. Paxton EW, Namba RS, Maletis GB, Khatod M, Yue EJ, Davies M et al (2010) A prospective study of 80,000 total joint and 5000 anterior cruciate ligament reconstruction procedures in a community-based registry in the United States. J Bone Joint Surg Am 92(Suppl 2):117-132

6. Moroni L, Fornasari PM (2013) Human mesenchymal stem cells: a bank perspective on the isolation, characterization and potential of alternative sources for the regeneration of musculoskeletal tissues. J Cell Physiol 228:680-687

7. Borakati A, Mafi R, Mafi P, Khan W (2017) A systematic review and meta-analysis of clinical trials of mesenchymal stem cell therapy for cartilage repair. Curr Stem Cell Res Ther. https://doi. org/10.2174/1574888X12666170915120620

8. Lv FJ, Tuan RS, Cheung KM, Leung VY (2014) Concise review: the surface markers and identity of human mesenchymal stem cells. Stem Cells 32:1408-1419

9. European Union (2009) Commission Directive 2009/120/EC of 14 September 2009 amending Directive 2001/83/EC of the European Parliament and of the Council on the Community code relating to medicinal products for human use as regards advanced therapy medicinal products. OJ L 242:3-12

10. European Union (2007) Regulation (EC) No 1394/2007 of the European Parliament and of the Council of 13 November 200 on advanced therapy medicinal products and amending. Directive 2001/83/EC and Regulation (EC) No 726/2004. OJ L 324:121-37

11. Vangsness CT Jr, Farr J 2nd, Boyd J, Dellaero DT, Mills CR, LeRoux-Williams M (2014) Adult human mesenchymal stem cells delivered via intra-articular injection to the knee following partial medial meniscectomy: a randomized, double-blind, controlled study. J Bone Joint Surg Am 96:90-98

12. Zhang J, Huang X, Wang H, Liu X, Zhang T, Wang $Y$ et al (2015) The challenges and promises of allogeneic mesenchymal stem cells for use as a cell-based therapy. Stem Cell Res Ther 6:234

13. Atesok K, Fu FH, Sekiya I, Stolzing A, Ochi M, Rodeo SA (2017) Stem cells in degenerative orthopaedic pathologies: effects of aging on therapeutic potential. Knee Surg Sports Traumatol Arthrosc 25:626-636

14. Beane OS, Fonseca VC, Cooper LL, Koren G, Darling EM (2014) Impact of aging on the regenerative properties of bone marrow-, muscle-, and adipose-derived mesenchymal stem/stromal cells. PLoS One 9:e115963

15. Cianfarani F, Toietta G, Di Rocco G, Cesareo E, Zambruno G, Odorisio T (2013) Diabetes impairs adipose tissue-derived stem cell function and efficiency in promoting wound healing. Wound Repair Regen 21:545-553

16. Sun Y, Deng W, Geng L, Zhang L, Liu R, Chen W et al (2015) Mesenchymal stem cells from patients with rheumatoid arthritis display impaired function in inhibiting Th17 cells. J Immunol Res 2015:284215

17. de Windt TS, Vonk LA, Slaper-Cortenbach IC, van den Broek MP, Nizak R, van Rijen MH et al (2017) Allogeneic mesenchymal stem cells stimulate cartilage regeneration and are safe for single-stage cartilage repair in humans upon mixture with recycled Autologous Chondrons. Stem Cells 35:256-264

18. de Windt TS, Vonk LA, Slaper-Cortenbach ICM, Nizak R, van Rijen MHP, Saris DBF (2017) Allogeneic MSCs and 
Recycled Autologous Chondrons mixed in a one-stage cartilage cell transplantion: a first-in-man trial in 35 patients. Stem Cells 35:1984-1993

19. Davatchi F, Abdollahi BS, Mohyeddin M, Shahram F, Nikbin B (2011) Mesenchymal stem cell therapy for knee osteoarthritis. Preliminary report of four patients. Int J Rheum Dis 14:211-215

20. Emadedin M, Aghdami N, Taghiyar L, Fazeli R, Moghadasali $\mathrm{R}$, Jahangir $\mathrm{S}$ et al (2012) Intra-articular injection of autologous mesenchymal stem cells in six patients with knee osteoarthritis. Arch Iran Med 15:422-428

21. Orozco L, Munar A, Soler R, Alberca M, Soler F, Huguet M et al (2013) Treatment of knee osteoarthritis with autologous mesenchymal stem cells: a pilot study. Transplantation 95:1535-1541

22. Orozco L, Munar A, Soler R, Alberca M, Soler F, Huguet M et al (2014) Treatment of knee osteoarthritis with autologous mesenchymal stem cells: two-year follow-up results. Transplantation 97:e66-e68

23. Emadedin M, Ghorbani Liastani M, Fazeli R, Mohseni F, Moghadasali R, Mardpour S et al (2015) Long-term follow-up of intraarticular injection of autologous mesenchymal stem cells in patients with knee, ankle, or hip osteoarthritis. Arch Iran Med $18: 336-344$

24. Vega A, Martin-Ferrero MA, Del Canto F, Alberca M, Garcia V, Munar A et al (2015) Treatment of knee osteoarthritis with allogeneic bone marrow mesenchymal stem cells: a randomized controlled trial. Transplantation 99:1681-1690

25. Soler R, Orozco L, Munar A, Huguet M, Lopez R, Vives J et al (2016) Final results of a phase I-II trial using ex vivo expanded autologous mesenchymal stromal cells for the treatment of osteoarthritis of the knee confirming safety and suggesting cartilage regeneration. Knee 23:647-654

26. Davatchi F, Abdollahi BS, Mohyeddin M, Nikbin B (2016) Mesenchymal stem cell therapy for knee osteoarthritis: 5 years followup of three patients. Int J Rheum Dis 19:219-225

27. Lamo-Espinosa JM, Mora G, Blanco JF, Granero-Molto F, NunezCordoba JM, Sanchez-Echenique C et al (2016) Intra-articular injection of two different doses of autologous bone marrow mesenchymal stem cells versus hyaluronic acid in the treatment of knee osteoarthritis: multicenter randomized controlled clinical trial (phase I/II). J Transl Med 14:246

28. Gupta PK, Chullikana A, Rengasamy M, Shetty N, Pandey V, Agarwal V et al (2016) Efficacy and safety of adult human bone marrow-derived, cultured, pooled, allogeneic mesenchymal stromal cells (Stempeucel(R)): preclinical and clinical trial in osteoarthritis of the knee joint. Arthritis Res Ther 18:301

29. Al-Najar M, Khalil H, Al-Ajlouni J, Al-Antary E, Hamdan M, Rahmeh R et al (2017) Intra-articular injection of expanded autologous bone marrow mesenchymal cells in moderate and severe knee osteoarthritis is safe: a phase I/II study. J Orthop Surg Res 12:190

30. Kim JD, Lee GW, Jung GH, Kim CK, Kim T, Park JH et al (2014) Clinical outcome of autologous bone marrow aspirates concentrate (BMAC) injection in degenerative arthritis of the knee. Eur J Orthop Surg Traumatol 24:1505-1511

31. Centeno C, Pitts J, Al-Sayegh H, Freeman M (2014) Efficacy of autologous bone marrow concentrate for knee osteoarthritis with and without adipose graft. Biomed Res Int 2014:370621

32. Centeno CJ, Al-Sayegh H, Bashir J, Goodyear S, Freeman MD (2015) A dose response analysis of a specific bone marrow concentrate treatment protocol for knee osteoarthritis. BMC Musculoskelet Disord 16:258

33. Sampson S, Smith J, Vincent H, Aufiero D, Zall M, Botto-vanBemden A (2016) Intra-articular bone marrow concentrate injection protocol: short-term efficacy in osteoarthritis. Regen Med $11: 511-520$
34. Shapiro SA, Kazmerchak SE, Heckman MG, Zubair AC, O'Connor MI (2017) A prospective, single-blind, placebo-controlled trial of bone marrow aspirate concentrate for knee osteoarthritis. Am J Sports Med 45:82-90

35. Jo CH, Lee YG, Shin WH, Kim H, Chai JW, Jeong EC et al (2014) Intra-articular injection of mesenchymal stem cells for the treatment of osteoarthritis of the knee: a proof-of-concept clinical trial. Stem Cells 32:1254-1266

36. Pers YM, Rackwitz L, Ferreira R, Pullig O, Delfour C, Barry F et al (2016) Adipose mesenchymal stromal cell-based therapy for severe osteoarthritis of the knee: a Phase I dose-escalation trial. Stem Cells Transl Med 5:847-856

37. Jo CH, Chai JW, Jeong EC, Oh S, Shin JS, Shim H et al (2017) Intra-articular injection of mesenchymal stem cells for the treatment of osteoarthritis of the knee: a 2-year follow-up study. Am J Sports Med 45:2774-2783

38. Song Y, Du H, Dai C, Zhang L, Li S, Hunter DJ et al (2018) Human adipose-derived mesenchymal stem cells for osteoarthritis: a pilot study with long-term follow-up and repeated injections. Regen Med 13:295-307

39. Pak J (2011) Regeneration of human bones in hip osteonecrosis and human cartilage in knee osteoarthritis with autologous adipose-tissue-derived stem cells: a case series. J Med Case Rep 5:296

40. Gibbs N, Diamond R, Sekyere EO, Thomas WD (2015) Management of knee osteoarthritis by combined stromal vascular fraction cell therapy, platelet-rich plasma, and musculoskeletal exercises: a case series. J Pain Res 8:799-806

41. Pak J, Lee JH, Park KS, Jeong BC, Lee SH (2016) Regeneration of cartilage in human knee osteoarthritis with autologous adipose tissue-derived stem cells and autologous extracellular matrix. Biores Open Access 5:192-200

42. Fodor PB, Paulseth SG (2016) Adipose derived stromal cell (ADSC) injections for pain management of osteoarthritis in the human knee joint. Aesthet Surg J 36:229-236

43. Bansal H, Comella K, Leon J, Verma P, Agrawal D, Koka P et al (2017) Intra-articular injection in the knee of adipose derived stromal cells (stromal vascular fraction) and platelet rich plasma for osteoarthritis. J Transl Med 15:141

44. Hudetz D, Boric I, Rod E, Jelec Z, Radic A, Vrdoljak T et al (2017) The effect of intra-articular injection of autologous microfragmented fat tissue on proteoglycan synthesis in patients with knee osteoarthritis. Genes (Basel). https://doi.org/10.3390/genes 8100270

45. Prockop DJ (2007) "Stemness" does not explain the repair of many tissues by mesenchymal stem/multipotent stromal cells (MSCs). Clin Pharmacol Ther 82:241-243

46. Caplan AI (2015) Adult mesenchymal stem cells: When, Where, and How. Stem Cells Int 2015:628767

47. Caplan AI (2016) MSCs: the sentinel and safe-guards of injury. J Cell Physiol 231:1413-1416

48. Crisan M, Yap S, Casteilla L, Chen CW, Corselli M, Park TS et al (2008) A perivascular origin for mesenchymal stem cells in multiple human organs. Cell Stem Cell 3:301-313

49. Caplan AI (2010) What's in a name? Tissue Eng Part A 16:2415-2417

50. Barry F, Murphy M (2013) Mesenchymal stem cells in joint disease and repair. Nat Rev Rheumatol 9:584-594

51. de Girolamo L, Kon E, Filardo G, Marmotti AG, Soler F, Peretti GM et al (2016) Regenerative approaches for the treatment of early OA. Knee Surg Sports Traumatol Arthrosc 24:1826-1835

52. Filardo G, Madry H, Jelic M, Roffi A, Cucchiarini M, Kon E (2013) Mesenchymal stem cells for the treatment of cartilage lesions: from preclinical findings to clinical application in orthopaedics. Knee Surg Sports Traumatol Arthrosc 21:1717-1729 
53. Tolar J, Le Blanc K, Keating A, Blazar BR (2010) Concise review: hitting the right spot with mesenchymal stromal cells. Stem Cells 28:1446-1455

54. Leijs MJ, van Buul GM, Lubberts E, Bos PK, Verhaar JA, Hoogduijn MJ et al (2012) Effect of arthritic synovial fluids on the expression of immunomodulatory factors by mesenchymal stem cells: an explorative in vitro study. Front Immunol 3:231

55. van Buul GM, Villafuertes E, Bos PK, Waarsing JH, Kops N, Narcisi R et al (2012) Mesenchymal stem cells secrete factors that inhibit inflammatory processes in short-term osteoarthritic synovium and cartilage explant culture. Osteoarthr Cartil 20:1186-1196

56. Manferdini C, Maumus M, Gabusi E, Piacentini A, Filardo G, Peyrafitte JA et al (2013) Adipose-derived mesenchymal stem cells exert antiinflammatory effects on chondrocytes and synoviocytes from osteoarthritis patients through prostaglandin E2. Arthritis Rheum 65:1271-1281

57. Topoluk N, Steckbeck K, Siatkowski S, Burnikel B, Tokish J, Mercuri J (2017) Amniotic mesenchymal stem cells mitigate osteoarthritis progression in a synovial macrophage-mediated in vitro explant coculture model. J Tissue Eng Regen Med. https ://doi.org/10.1002/term.2610

58. Bianchi F, Maioli M, Leonardi E, Olivi E, Pasquinelli G, Valente $S$ et al (2013) A new nonenzymatic method and device to obtain a fat tissue derivative highly enriched in pericyte-like elements by mild mechanical forces from human lipoaspirates. Cell Transpl 22:2063-2077

59. Friji MT (2014) Nanofat grafting: basic research and clinical applications. Plast Reconstr Surg 134:333e-334e

60. Vines JB, Aliprantis AO, Gomoll AH, Farr J (2016) Cryopreserved amniotic suspension for the treatment of knee osteoarthritis. J Knee Surg 29:443-450

61. Dhillon RS, Schwarz EM, Maloney MD (2012) Platelet-rich plasma therapy—future or trend? Arthritis Res Ther 14:219

62. Hyun JS, Tran MC, Wong VW, Chung MT, Lo DD, Montoro DT et al (2013) Enhancing stem cell survival in vivo for tissue repair. Biotechnol Adv 31:736-743

63. Hurley ET, Yasui Y, Gianakos AL, Seow D, Shimozono Y, Kerkhoffs $\mathrm{G}$ et al (2018) Limited evidence for adipose-derived stem cell therapy on the treatment of osteoarthritis. Knee Surg Sports Traumatol Arthrosc. https://doi.org/10.1007/s00167-018-4955-x

64. Nguyen LT, Sharma AR, Chakraborty C, Saibaba B, Ahn ME, Lee SS (2017) Review of prospects of biological fluid biomarkers in osteoarthritis. Int J Mol Sci. https://doi.org/10.3390/ijms180306 01 